\title{
Production and Nutritional Evaluation of Instantized Kunun Gyada, a Traditional Nigerian Beverage from Malted Sorghum (Sorghum bicolor) and Roasted Groundnut (Arachis hypogeae) Paste by Extrusion Cooking.
}

\author{
Ularamu, J.J. ${ }^{1}$, , Maina, J.F. ${ }^{2}$ Ta'awu, K.G ${ }^{3}$., and Nwankwo, R.N. ${ }^{4}$ \\ ${ }^{1}$ Department of Food Science and Technology, Federal Polytechnic Mubi, Adamawa State, Nigeria. \\ ${ }^{2}$ Department of Food Science and Technology, Federal Polytechnic Mubi, Adamawa State, Nigeria. \\ ${ }^{3}$ Department of Food Science and Technology, Federal Polytechnic Mubi, Adamawa State, Nigeria. \\ ${ }^{4}$ Department of Nutrition and Dietetics, Federal Polytechnic Mubi, Adamawa State, Nigeria.
}

\begin{abstract}
The effect of malting and extrusion on the chemical properties of Kunun Gyada extrudates from malted sorghum flour and roasted groundnut paste was investigated. 27 samples using full factorial design were processed in a co-rotating twin-screw extruder. The results were subjected to statistical analyses to test for significant differences between treatments. Analysis of variance (ANOVA) was adopted using Statistix 9; Version 9.1 (2012) Statistical Package. There was increase in proximate mean composition of germinated sorghum flour, moisture 11.20 to $11.23 \%$, ash 1.23 to $1.37 \%$, and crude protein 8.50 to $9.13 \%$ with decrease in both crude fat and carbohydrates from 3.50 to $3.17 \%$ and 77.70 to $74.27 \%$ respectively. Proximate composition of roasted groundnuts paste showed increase in crude fat and decrease in carbohydrates, moisture content, ash, and crude protein. Crude protein content of extrudates increased from $19.13 \%$ (malted sorghum) to $22.9 \%$. Feed moisture content increased from $0.4 \mathrm{~g} / \mathrm{ml}$ to $0.7 \mathrm{~g} / \mathrm{ml}$. Among all the amino acids at extrusion parameters $35 \%, 20 \%, 110^{\circ} \mathrm{C}$, Methionine had the lowest value of $1.63 \mathrm{~g}$ and Glutamic acid has the highest value of $17.28 \mathrm{~g}$. From essential amino acids, Methionine had the lowest value of $1.63 \mathrm{~g}$, Leucine had the highest value of $7.34 \mathrm{~g}$ and lysine had $3.54 \mathrm{~g}$ at the same design point $23\left(35 \% 20 \% 110^{\circ} \mathrm{C}\right)$. In non-essential amino acids, Cystine had the lowest value of $2.40 \mathrm{~g}$ and Glutamic acid had the highest value of $17.28 \mathrm{~g}$ at $35 \% 20 \% 110^{\circ} \mathrm{C}$. The results of proximate mean composition and amino acid profile contents of extrudates indicates increase in nutrients which may be as a result of the increase in roasted groundnuts paste, use of malted sorghum flour, and the application of extrusion cooking which enhances the nutrient densities of cereal gruels and improve the product quality. The extrudate with design point 20 for its high protein content and required essential amino acid values has better nutritional potential for commercial production of kunungyada in Nigeria.
\end{abstract}

Keyword: Extrusion, Malting, Roasting, Kunungyada, Sorghum.

\section{Introduction}

Cereals form an important part of the diet of many people in the developing countries especially in Nigeria. These include maize, sorghum, millet, and rice. Cereals are associated with food and drinks throughout the history of Mankind. This close association with people's lives may have been because cereals are easy to cultivate, transport, store, and are adoptable to different environment. Cereals are used mostly as foods and feeds (Gibson et al., 1998). Cereals are also used for making porridges and starch based meals which are used for breakfast formulations. The outlook for world cereal production in 2015 has improved since the previous report in May, on expectations of larger wheat, coarse grains (maize, sorghum and millet) and rice harvests. FAO's latest forecast for global cereal production in 2015 stands at 2524 million tonnes almost 15 million tonnes higher than it was reported in May. At this level, world cereal production will be 1 percent or 25.6 million tonnes, lower than the record in 2014 (FAO, 2015).

Sorghum is a cereal grain widely grown across the world. Sorghum survives draught condition better than maize and other cereals. It is commonly grown in areas where rainfall is low and unpredictable especially in the savannah grassland in Nigeria. It is a valuable food crop because it contains a higher percentage of protein than maize and the protein is also of better quality, with respect to amino acid profile. Sorghum is also rich in calcium and iron. In some parts of Asia and Africa it is regarded as low-class foods for poor people (Zhang and Harmaker, 1998). World Sorghum Production 2015/2016, June 2015, the United States Department of Agriculture (USDA) estimates that the World Sorghum production will be 65.34 million metric tonnes, around 0.06 metric tonnes less than the previous month's projection. Sorghum production in 2015 was 64.08 million tonnes. 2015's 65.34 estimated million tonnes could represent an increase of 1.24 million tonnes or a $1.94 \%$ in sorghum production around the world. Sorghum production by country; United States is leading with 11,050,000 metric tonnes, followed by Mexico with 7, 800,000 metric tonnes, Nigeria 6,150,000 metric tonnes, 
Production and Nutritional Evaluation of Instantized Kunun Gyada, a Traditional Nigerian ..

Sudan 5,500,000 metric tonnes, India 5,500,000 metric tonnes, Argentina 4,500,000 metric tonnes, Ethiopia 4,000,000 metric tonnes, China 2,600,000 metric tonnes, Australia 2,100,000 metric tonnes, Brazil 2,000,000 metric tonnes, Burkina Faso 1,900,000 metric tonnes, Mali 1,300,000 metric tonnes, Cameroun 1, 150,000 metric tonnes, Niger 1,100,000 metric tonnes, Chad 900,000 metric tonnes and Tanzania 840,000 metric tonnes (USDA, 2015).

Groundnut also known as peanut or monkey-nut is specie in the legume family (Fabaceae). In West Africa, groundnut grows well in Southern Mali and adjacent regions of the Ivory Coast, Burkina Faso, Ghana, Nigeria and Senegal (Gordon, 2001). They are energy dense foods because of their oil, and they are rich in vitamins and minerals (Eke-Ejiofor et al., 2012). Foods containing roasted groundnuts have high consumer acceptance because of their unique roasted peanut flavour. Peanut are continually applied for the preparation of new and improved food products (Eke-Ejiofor et al., 2012).

Extrusion cooking is a high- temperature, shot-time process in which moistened, expansive, starchy and/or protenacious food materials are plasticised and cooked in a tube by a combination of moisture, pressure, temperature and mechanical shear, resulting in molecular transformation and chemical reactions (Castells et al., 2005). This technology has some unique positive features compared with other heat processes; the material is subjected to intense mechanical shear. Extrusion cooking is preferable to other food processing techniques in terms of continuous process with high productivity and significant nutrient retention, owing to high temperature and short time required (Guy, 2001). Nutritional concern about extrusion cooking is reached at its highest level when extrusion is used specifically to produce nutritionally balanced or enriched foods (Plahar et al., 2003).

To improve the nutrient intake of communities in Africa especially children, several food preparations technology have been advocated that can effectively increase the nutrient density of porridges and reduce the risk of malnutrition. The process has found numerous applications, including increasing numbers of ready-to-eat cereals; salty and sweet snacks; co-extruded snacks; indirect expanded products; an expanded array of dry pet foods and fish foods; textured meat-like materials from defatted high-protein flours; nutritious precooked food mixtures for infant feeding; and confectionery products (Eastman et al., 2001).

The current varieties of food products obtained through extrusion cooking are impressive and continue to expand (Harper, 1984). There is possibility of extrusion cooking of mixed malted sorghum flour and roasted groundnuts paste to produce kunun gyada, this will provide a useful alternative in highly nutritious food products and improve on the traditional methods of preparation which form the thrust of this work.

\section{Materials And Methods}

\section{Malting of Sorghum}

Sorghum grains (Sorghum bicolor) obtained from Kasuwan Kuturu in Mubi, Adamawa State, Nigeria, were cleaned and germinated using the method of Elkhalita and Bernhardt, (2010). The sorghum grains were soaked in clean water for 24 hours at room temperature $\left(30^{\circ} \mathrm{C}\right)$ with two changes of water to ensure removal of dirt and other contaminants. The carefully drained soaked grains were spread out on a jute bag saturated with water on a clean floor covered with another jute bag under shade for two days at room temperature $\left(25-30^{\circ} \mathrm{C}\right)$ and water was sprayed on the grains after every six hours to control the moisture level. The germinated grains were sundried for three days at temperatures of about $35-40^{\circ} \mathrm{C}$.

\section{Flour Preparation}

The root and shoot portions were manually removed by gently pounding using pestle and mortar. The polished dried malted sorghum grains were ground to fine homogenous powder using a laboratory mill (SPI Supplies PA, USA) and passed through a $100 \mu \mathrm{m}$ mesh screen. The milled sample was packed in a plastic container and stored at low temperature until required for use.

\section{Paste Preparation}

The groundnuts (Arachis Hypogeae) variety Virginia bunch was purchased in Kasuwan Kuturu in Mubi, Adamawa state, Nigeria. The kernels were red, uniform, and medium in size. After sorting, cleaning and grading, it was mildly roasted using clean heated dry sand at about $98^{\circ} \mathrm{C}$ in a clay pot. The roasted groundnut was blanched (removing the husk) by robbing between two clean wooden surfaces to break and remove the husk from the groundnut seeds and winnowed to separate the husk from the seeds. The cleaned groundnuts were milled into a paste of golden brown colour using disc attrition mill.

\section{Blend Preparation and Moisture Adjustment}

One thousand grams weight blend of samples were obtained by mixing the given percentage of groundnuts paste in each with the sorghum malt flour and the total moisture content of blends were determined on wet weight basis (Filli et al. 2011). 
Production and Nutritional Evaluation of Instantized Kunun Gyada, a Traditional Nigerian ..

\section{Experimental Design}

A three- factor three levels full factorial design was adopted for the study to determine the physicochemical and sensory properties of extrudates from the blends of malted sorghum flour and roasted groundnuts paste based kunu gyada (Filli et al., 2010). Details of the Experimental Design in their Coded and Natural form are shown on Tables 1 and 2.

\section{Extrusion Cooking}

The extrusion cooking was performed using SLG65 Twin Screw Extruder, Jinansaibainuo Technology Development Co; LMD in The Federal Polytechnic Mubi, Adamawa State, Nigeria. The extruder has three independent heating zones and the samples were extruded at 100,110 and $120^{\circ} \mathrm{C}$ respectively. The length to diameter (L/D) of the extruder was 20:1. The diameter of the die was $4 \mathrm{~mm}$ with die length of $27 \mathrm{~mm}$. The moisture content of different blends was adjusted by addition of a predetermined amount of water to 15,20 and $25 \%$ respectively. The extruder was fed manually through a screw operated conical hopper.

\section{Proximate Analysis}

Unmalted and malted sorghum flours, unroasted and roasted groundnut pastes, and ground samples of extrudates for proximate composition (protein, amino acid profile, fat, carbohydrates, ash, and moisture) were analysed using these procedures. Protein was determined by Kjeldhal method using KDN-2C Nitrogen/Protein determinator. Percentage nitrogen was converted into crude protein by multiplying with the conversion factor 6.25. The method of Blamire (2003) was adopted for the calculation of the crude protein. The fat content was analysed using the methods described by (AOAC 2000). The carbohydrates was determined by difference $100-$ [protein $(\%)+$ fat $(\%)+$ moisture $(\%)+$ ash (\%)] as described by Egan et al, (1981). The amino acid profile in the known extrudate samples was determined using methods described by Benitez (1989). The amount of each amino acid present in the sample was calculated in $\mathrm{g} / 16 \mathrm{gN}$ or $\mathrm{g} / 100 \mathrm{~g}$ protein. Ash content was determined using the methods described by Onwuka, (2005). The moisture content was determined as described by AOAC (2006).

\section{Statistical Analysis}

The results were subjected to statistical analyses to test for significant differences between treatments. Statistix 9, version 9.1 (2012) Statistical package was used. ANOVA was adopted to test for significant difference at $\mathrm{p}<$ 0.05 level of significance for the sensory evaluation. Values were means of triplicate analyses.

\section{Proximate Composition of the Raw Materials}

\section{Results}

The results of proximate compositions of the raw materials are presented in Tables 3 and 4 . The protein content of unroasted groundnuts $27.8 \%$ showed a value higher than roasted groundnuts $26.9 \%$, similarly fat content increased from $49 \%$ to 53\%. Carbohydrates contents decreased from $20.6 \%$ to $19.3 \%$. Moisture level decreased from $3.8 \%$ to $2.2 \%$ and the ash content also decreased from $2.7 \%$ in unroasted groundnuts to $2.5 \%$. The protein content of germinated sorghum flour $9 \%$ was higher than the non-germinated sorghum flour $8.4 \%$. The fat content was higher in non- germinated sorghum flour $3.3 \%$ than the germinated sorghum flour $3.2 \%$. Flour from germinated sorghum had slightly higher moisture content $11.2 \%$ than non-germinated sorghum flour $11.1 \%$. The germinated sorghum flour had higher ash content $1.4 \%$ than the non-germinated sorghum $1.2 \%$. While the carbohydrates content of unmalted sorghum flour was $77.6 \%$ compared to $74.3 \%$ in the malted sorghum.

\section{Proximate Composition of Extrudates}

The mean values of the proximate composition of extrudates are presented in Table 5. The crude protein content values increased from $9.13 \%$ for sorghum malt to $16.06 \%$ and $22.89 \%$ for design points 11 and 20 respectively with increase in feed composition from 15 to $35 \%$ and decrease in barrel temperature from $120^{\circ} \mathrm{C}$ to $110^{\circ} \mathrm{C}$ as the level of enrichment with roasted groundnuts paste increased. The fat values increased from 6 to 23\%, the result showed that increase in both feed composition (15 to 35\%) and barrel temperature $\left(110\right.$ to $120^{\circ} \mathrm{C}$ ) increased the fat content. The moisture content of extrudates varies between 3.17 to $5.50 \%$. The ash content of the extrudates reduced from 3 to $1 \%$ with sample formulation ratios of $15 \%$ feed composition, $15 \%$ moisture content, $120^{\circ} \mathrm{C}$ barrel temperature and $35 \%$ feed composition, $25 \%$ moisture content, $120^{\circ} \mathrm{C}$ barrel temperature. The carbohydrates contents of the extrudates reduced from 72 to $53 \%$ with sample formulation ratios of $15 \%$ feed composition, $15 \%$ moisture content, $110^{\circ} \mathrm{C}$ barrel temperature and $35 \%$ feed composition, $25 \%$ moisture content, $110^{\circ} \mathrm{C}$ barrel temperature respectively. 
Production and Nutritional Evaluation of Instantized Kunun Gyada, a Traditional Nigerian ..

Table 1. Proximate Composition of Non-germinated and Germinated Sorghum Flour (\%)

\begin{tabular}{|l|l|l|}
\hline Composition & Non-germinated sorghum & Germinated sorghum \\
\hline Moisture & $11.20 \pm 0.10 \mathrm{a}$ & $11.023 \pm 0.06 \mathrm{a}$ \\
\hline Ash & $1.23 \pm 0.06 \mathrm{a}$ & $1.37 \pm 0.06 \mathrm{~b}$ \\
\hline Crude protein & $8.50 \pm 0.10 \mathrm{a}$ & $9.13 \pm 0.06 \mathrm{~b}$ \\
\hline Crude fat & $3.50 \pm 0.10 \mathrm{a}$ & $3.17 \pm 0.06 \mathrm{~b}$ \\
\hline Carbohydrates & $77.70 \pm 0.10 \mathrm{a}$ & $74.27 \pm 0.06 \mathrm{~b}$ \\
\hline
\end{tabular}

Any two means in the same column followed by the same letter are not significantly different $(\mathrm{p}>0.05)$.

Table 2. Proximate Composition of Raw and Roasted Groundnuts Paste (\%)

\begin{tabular}{|l|l|l|}
\hline Composition & Raw groundnuts paste & Roasted groundnuts paste \\
\hline Moisture & $3.77 \pm 0.06^{\mathrm{a}}$ & $2.30 \pm 0.10^{\mathrm{b}}$ \\
\hline Ash & $2.63 \pm 0.12^{\mathrm{a}}$ & $2.60 \pm 0.10^{\mathrm{a}}$ \\
\hline Crude protein & $27.77 \pm 0.15^{\mathrm{a}}$ & $26.80 \pm 0.10^{\mathrm{b}}$ \\
\hline Fats & $49.33 \pm 1.53^{\mathrm{a}}$ & $53.33 \pm 1.53^{\mathrm{b}}$ \\
\hline Carbohydrates & $20.73 \pm 0.15^{\mathrm{a}}$ & $19.40 \pm 0.10^{\mathrm{b}}$ \\
\hline
\end{tabular}

Any two means in the same column followed by the same letter are not significantly $(\mathrm{p}>0.05)$.

Table3, Experimental Design and Observed Values of Proximate Mean Composition of Extrudates (\%)

\begin{tabular}{|c|c|c|c|c|c|c|c|c|}
\hline 1 & 15 & 15 & 100 & $17.29 \pm 0.04 \mathrm{Im}$ & $11.00=1.021$ & $66.71 \pm 0.61 j$ & $2.00=0.04 \mathrm{~g}$ & $3.03=1.02 \mathrm{~b}$ \\
\hline 2 & 15 & 15 & 110 & $16.98=1.02 \mathrm{~m}$ & $7.00=0.04 \mathrm{op}$ & $72.00 \pm 1.01 \mathrm{a}$ & $1.00 \pm 0.03 \mathrm{~g}$ & $3.00 \pm 0.02 c$ \\
\hline 3 & 15 & 15 & 120 & $16.67 \pm 0.040$ & $7.33 \pm 0.02 n$ & $67.50 \pm 0.02 \mathrm{~b}$ & $3.00 \pm 0.05 \mathrm{~h}$ & $4.50 \pm 0.02 \mathrm{a}$ \\
\hline 4 & 15 & 20 & 100 & $17.04=1.02 \mathrm{~m}$ & $12.00 \pm 1.02 \mathrm{k}$ & $66.90=0.01 \mathrm{i}$ & $1.00 \pm 0.02 \mathrm{~g}$ & $3.00 \pm 0.01 \mathrm{c}$ \\
\hline 5 & 15 & 20 & 110 & $16.49=0.07 n$ & $11.33 \pm 0.031$ & $67.68=0.05 \mathrm{~h}$ & $2.00 \pm 5.77 \mathrm{f}$ & $3.50 \pm 0.06 \mathrm{~b}$ \\
\hline 6 & 15 & 20 & 120 & $17.70 \pm 0.02 \mathrm{k}$ & $9.60 \pm 1.00 \mathrm{~m}$ & $67.10 \pm 0.10 \mathrm{f}$ & $2.00 \pm 0.60 \mathrm{i}$ & $3.17 \pm 1.02 \mathrm{~b}$ \\
\hline 7 & 15 & 25 & 100 & $18.60 \pm 0.06 \mathrm{ij}$ & $8.00 \pm 0.03 n$ & $67.90 \pm 0.06 \mathrm{~g}$ & $1.00 \pm 0.27 \mathrm{~cd}$ & $4.67 \pm 0.01 \mathrm{c}$ \\
\hline 8 & 15 & 25 & 110 & $17.32 \pm 1.02 \mathrm{klm}$ & $6.00 \neq 1.02 q$ & $70.17 \pm 0.01 \mathrm{~d}$ & $2.00 \pm 1.02 \mathrm{a}$ & $5.50 \pm 1.02 \mathrm{~b}$ \\
\hline 9 & 15 & 25 & 120 & $17.51 \pm 0.04 \mathrm{kl}$ & $6.67 \pm 0.03 p$ & $69.32 \pm 0.03 \mathrm{e}$ & $2.00 \pm 0.03 \mathrm{~d}$ & $4.50 \pm 0.03 \mathrm{~b}$ \\
\hline 10 & 25 & 15 & 100 & $22.27 \pm 0.15 b$ & $15.33 \pm 0.12 \mathrm{~h}$ & $57.85 \pm 0.05 \mathrm{~s}$ & $0.99 \pm 1.02 \mathrm{f}$ & $3.50 \pm 0.03 c$ \\
\hline 11 & 25 & 15 & 110 & $16.06 \pm 0.030$ & $8.33=0.2 \ln$ & $71.11=0.01 \mathrm{c}$ & $1.00=0.09 \mathrm{f}$ & $3.50 \pm 0.02 c$ \\
\hline 12 & 25 & 15 & 120 & $20.32 \pm 1.02 \mathrm{e}$ & $15.00 \pm 0.85 \mathrm{a}$ & $49.15 \pm 0.04 z$ & $2.00 \pm 0.03 \mathrm{f}$ & $3.50 \pm 0.02 b$ \\
\hline 13 & 25 & 20 & 100 & $21.11 \neq 0.02 \mathrm{~cd}$ & $15.00 \pm 0.04 \mathrm{~h}$ & $58.40 \pm 5.77 \mathrm{r}$ & $2.00 \pm 0.02 \mathrm{f}$ & $3.50 \pm 0.02 b$ \\
\hline 14 & 25 & 20 & 110 & $18.82 \pm 0.06 \mathrm{ghi}$ & $16.33 \pm 0.02 \mathrm{~g}$ & $68.85 \pm 0.11 q$ & $2.00 \pm 0.08 \mathrm{e}$ & $4.00 \pm 1.02 b$ \\
\hline 15 & 25 & 20 & 120 & $21.89=0.02 \mathrm{~b}$ & $13.00 \pm 0.02 j$ & $60.61=0.07 \%$ & $1.00 \pm 0.05 \mathrm{f}$ & $3.50 \pm 0.03 c$ \\
\hline 16 & 25 & 25 & 100 & $18.62 \pm 0.11 \mathrm{hij}$ & $13.33 \pm 0.24 \mathrm{ij}$ & $62.55 \pm 0.02 \mathrm{n}$ & $1.00 \pm 1.02 \mathrm{~d}$ & $4.50 \pm 0.04 \mathrm{c}$ \\
\hline 17 & 25 & 25 & 110 & $19.03 \pm 1.02 \mathrm{gh}$ & $12.00 \pm 0.02 \mathrm{k}$ & $62.97 \pm 0.111 \mathrm{~m}$ & $1.00 \pm 0.32 \mathrm{bc}$ & $4.83 \pm 0.07 \mathrm{c}$ \\
\hline 18 & 25 & 25 & 120 & $19.18 \pm 0.16 \mathrm{fg}$ & $10.00=0.04 \mathrm{~m}$ & $64.33 \pm 0.21 \mathrm{k}$ & $1.00 \pm 0.27 \mathrm{a}$ & $5.33 \neq 0.03 \mathrm{c}$ \\
\hline 19 & 35 & 15 & 100 & $21.42 \pm 0.10 \mathrm{c}$ & $13.67 \pm 0.34 \mathrm{i}$ & $60.34 \pm 0.19 p$ & $1.00 \pm 1.02 \mathrm{f}$ & $3.50 \pm 0.08 \mathrm{c}$ \\
\hline 20 & 35 & 15 & 110 & $22.89=0.72 \mathrm{a}$ & $18.00 \pm 0.13 \mathrm{f}$ & $54.61 \pm 0.11 \mathrm{u}$ & $1.00=0.09 \mathrm{f}$ & $3.53 \pm 0.07 \mathrm{c}$ \\
\hline 21 & 35 & 15 & 120 & $18.21 \neq 0.11 \mathrm{j}$ & $23.00 \pm 0.04 b$ & $54.29 \pm 0.21 \mathrm{v}$ & $1.00 \pm 0.02 \mathrm{f}$ & $3.50 \pm 0.04 \mathrm{c}$ \\
\hline 22 & 35 & 20 & 100 & $17.16 \pm 0.02 \mathrm{~lm}$ & $18.66 \pm 0.27 \mathrm{e}$ & $58.17 \pm 0.19 \mathrm{r}$ & $2.00 \pm 0.06 \mathrm{e}$ & $4.00 \pm 0.09 \mathrm{~b}$ \\
\hline 23 & 35 & 20 & 110 & $18.25 \pm 0.14 \mathrm{j}$ & $13.67 \pm 0.14 i$ & $59.08=0.091$ & $1.00 \pm 0.07 \mathrm{f}$ & $3.00 \pm 0.05 c$ \\
\hline 24 & 35 & 20 & 120 & $18.61 \pm 1.02 \mathrm{ij}$ & $15.00 \pm 0.22 \mathrm{~h}$ & $62.90 \pm 1.02 \mathrm{~m}$ & $1.00 \pm 0.03 \mathrm{f}$ & $4.50 \pm 0.09 \mathrm{c}$ \\
\hline 25 & 35 & 25 & 100 & $18.43 \pm 0.22 \mathrm{ij}$ & $20.67 \pm 0.12 d$ & $53.8 \pm 0.10 \mathrm{w}$ & $2.00 \pm 0.08 \mathrm{~b}$ & $5.00 \pm 0.03 b$ \\
\hline 26 & 35 & 25 & 110 & $20.83 \pm 0.12 \mathrm{~d}$ & $21.67 \pm 0.34 \mathrm{c}$ & $53.00 \pm 1.02 \mathrm{x}$ & $1.00 \pm 0.04 \mathrm{~h}$ & $3.50 \pm 0.05 c$ \\
\hline 27 & 35 & 25 & 120 & $19.52=0.21 \mathrm{f}$ & $18.67 \pm 0.09 \mathrm{e}$ & $56.80 \pm 0.02 t$ & $1.00 \pm 0.02 \mathrm{e}$ & $4.00=0.07 \mathrm{c}$ \\
\hline
\end{tabular}

Table 4. Experimental Design and Observed Mean Values of Essential Amino Acids Profile of Extrudates

\begin{tabular}{|c|c|c|c|c|c|c|c|c|c|c|c|c|}
\hline $\begin{array}{l}\text { Design point } \\
\end{array}$ & $\mathrm{X}_{1}$ & $\mathrm{X}_{2}$ & $\mathrm{X}_{3}$ & Arginine & Histidine & Isoleucine & Leucine & Lysine & Methionine & Phenylalanine & Threonine & Valine \\
\hline 2 & 15 & 15 & 110 & $6.79 \pm 0.020$ & $2.22 \pm 0.04 \mathrm{~b}$ & $3.04 \pm 0.03 \mathrm{k}$ & $5.62 \pm 0.02 \mathrm{gh}$ & $3.15 \pm 0.03 \mathrm{jk}$ & $1.09 \pm 0.04 \mathrm{~b}$ & $3.96 \pm 0.02 \mathrm{klm}$ & $2.04 \pm 0.03 \mathrm{mn}$ & $3.57 \pm 0.02 \mathrm{~lm}$ \\
\hline 3 & 15 & 15 & 120 & $6.53 \pm 0.04 \mathrm{r}$ & $2.08 \pm 0.03 \mathrm{~b}$ & $3.00 \pm 0.03 \mathrm{k}$ & $5.42 \pm 0.03 \mathrm{hi}$ & $3.14 \pm 0.02 \mathrm{k}$ & $1.06 \pm 0.02 \mathrm{~b}$ & $3.93 \pm 0.03 \mathrm{klm}$ & $2.00 \pm 0.03 \mathrm{no}$ & $3.54 \pm 0.02 \mathrm{~m}$ \\
\hline 5 & 15 & 20 & 110 & $6.22 \pm 0.02 \mathrm{t}$ & $2.04 \pm 0.03 \mathrm{~b}$ & $2.72 \pm 0.03 \mathrm{n}$ & $5.09 \pm 0.03 \mathrm{ijkl}$ & $3.00 \pm 0.04 \mathrm{mn}$ & $1.05 \pm 0.02 \mathrm{~b}$ & $3.82 \pm 0.03 \mathrm{~m}$ & $1.95 \pm 0.03 p$ & $3.26 \pm 0.020$ \\
\hline 6 & 15 & 20 & 120 & $6.06 \pm 0.03 \mathrm{u}$ & $1.99 \pm 0.03 \mathrm{~b}$ & $2.68 \pm 0.030$ & $5.04 \pm 0.03 \mathrm{jkl}$ & $2.97 \pm 0.02 \mathrm{no}$ & $1.03 \pm 0.04 \mathrm{~b}$ & $3.75 \pm 0.04 \mathrm{n}$ & $1.94 \pm 0.04 p$ & $3.23 \pm 0.04 \mathrm{op}$ \\
\hline 7 & 15 & 25 & 100 & $5.69 \pm 0.02 \mathrm{v}$ & $1.98 \pm 0.05 \mathrm{~b}$ & $2.55 \pm 0.02 p$ & $4.98 \pm 0.03 \mathrm{kl}$ & $2.96 \pm 0.01$ no & $0.98 \pm 0.03 b$ & $3.67 \pm 0.020$ & $1.89 \pm 0.03 q$ & $3.20 \pm 0.04 \mathrm{pq}$ \\
\hline 8 & 15 & 25 & 110 & $5.44 \pm 0.03 \mathrm{w}$ & $7.69 \pm 9.95 \mathrm{a}$ & $2.50 \pm 0.03 q$ & $4.27 \pm 1.17 \mathrm{~m}$ & $2.96 \pm 0.03 \mathrm{no}$ & $0.89 \pm 0.02 b$ & $3.56 \pm 0.02 p$ & $1.84 \pm 0.02 \mathrm{r}$ & $3.15 \pm 0.02 q$ \\
\hline 9 & 15 & 25 & 120 & $5.10 \pm 0.03 x$ & $1.84 \pm 0.03 \mathrm{~b}$ & $2.43 \pm 0.03 \mathrm{r}$ & $4.87 \pm 0.041$ & $2.94 \pm 0.030$ & $0.84 \pm 0.03 \mathrm{~b}$ & $3.47 \pm 0.01 \mathrm{q}$ & $1.78 \pm 0.03 \mathrm{~s}$ & $3.15 \pm 0,02 q$ \\
\hline 13 & 25 & 20 & 100 & $7.33 \pm 0.02 \mathrm{k}$ & $2.19 \pm 0.03 \mathrm{~b}$ & $3.07 \pm 0.02 \mathrm{jk}$ & $5.87 \pm 0.02 \mathrm{fg}$ & $3.19 \pm 0.03 \mathrm{ij}$ & $1.17 \pm 0.03 \mathrm{~b}$ & $4.06 \pm 0.02 \mathrm{i}$ & $2.23 \pm 0.03 \mathrm{k}$ & $3.80 \pm 0.02 \mathrm{i}$ \\
\hline 14 & 25 & 20 & 110 & $6.65 \pm 0.02 q$ & $2.10 \pm 0.03 \mathrm{~b}$ & $2.99 \pm 0.031$ & $5.36 \pm 0.02 \mathrm{hij}$ & $3.13 \pm 0.07 \mathrm{k}$ & $1.14 \pm 0.08 \mathrm{~b}$ & $3.99 \pm 0.02 \mathrm{j}$ & $2.12 \pm 0.031$ & $3.58 \pm 0.08 \mathrm{kl}$ \\
\hline 15 & 25 & 20 & 120 & $6.70 \pm 0.03 \mathrm{p}$ & $2.13 \pm 0.04 \mathrm{~b}$ & $2.99 \pm 0.031$ & $5.55 \pm 0.02 \mathrm{gh}$ & $3.06 \pm 0.031$ & $1.14 \pm 0.03 \mathrm{~b}$ & $3.99 \pm 0.04 \mathrm{k}$ & $2.29 \pm 0.02 j$ & $3.57 \pm 0.02 \mathrm{~lm}$ \\
\hline 16 & 25 & 25 & 100 & $6.86 \pm 0.02 \mathrm{n}$ & $2.14 \pm 0.02 \mathrm{~b}$ & $3.04 \pm 0.03 \mathrm{k}$ & $6.00 \pm 0.03 \mathrm{ef}$ & $3.04 \pm 0.03 \mathrm{~lm}$ & $1.20 \pm 0.03 \mathrm{~b}$ & $3.99 \pm 0.04 \mathrm{k}$ & $2.47 \pm 0.02 \mathrm{i}$ & $3.59 \pm 0.02 \mathrm{kl}$ \\
\hline 17 & 25 & 25 & 110 & $6.93 \pm 0.02 \mathrm{~m}$ & $2.14 \pm 0.03 \mathrm{~b}$ & $3.10 \pm 0.02 \mathrm{ij}$ & $6.30 \pm 0.03 \mathrm{de}$ & $3.00 \pm 0.03 \mathrm{mn}$ & $1.29 \pm 0.02 \mathrm{~b}$ & $3.96 \pm 0.01 \mathrm{klm}$ & $2.70 \pm 0.03 \mathrm{~g}$ & $3.63 \pm 0.03 \mathrm{jk}$ \\
\hline 18 & 25 & 25 & 120 & $7.00 \pm 0.021$ & $2.15 \pm 0.03 \mathrm{~b}$ & $3.13 \pm 0.02 \mathrm{i}$ & $6.73 \pm 0.02 \mathrm{bc}$ & $2.98 \pm 0.01 \mathrm{no}$ & $1.36 \pm 0.03 \mathrm{~b}$ & $3.98 \pm 0.03 \mathrm{kl}$ & $2.83 \pm 0.03 \mathrm{f}$ & $3.67 \pm 0.02 \mathrm{j}$ \\
\hline 19 & 35 & 15 & 100 & $8.55 \pm 0.01 \mathrm{f}$ & $2.29 \pm 0.03 \mathrm{~b}$ & $3.44 \pm 0.02 \mathrm{f}$ & $7.12 \pm 0.03 \mathrm{a}$ & $3.14 \pm 0.03 \mathrm{k}$ & $1.47 \pm 0.03 \mathrm{~b}$ & $4.49 \pm 0.03 \mathrm{~g}$ & $2.99 \pm 0.03 \mathrm{e}$ & $4.13 \pm 0.02 \mathrm{f}$ \\
\hline 20 & 35 & 15 & 110 & $8.70 \pm 0.03 \mathrm{e}$ & $2.34 \pm 0.03 \mathrm{~b}$ & $3.50 \pm 0.03 \mathrm{e}$ & $7.20 \pm 0.03 \mathrm{a}$ & $3.23 \pm 0.03 \mathrm{hi}$ & $1.53 \pm 0.02 \mathrm{~b}$ & $4.49 \pm 0.03 \mathrm{e}$ & $3.07 \pm 0.01 \mathrm{~d}$ & $4.27 \pm 0.01 \mathrm{e}$ \\
\hline 21 & 35 & 15 & 120 & $9.07 \pm 0.02 \mathrm{~d}$ & $2.37 \pm 0.02 \mathrm{~b}$ & $3.59 \pm 0.03 \mathrm{~d}$ & $7.27 \pm 0.01 \mathrm{a}$ & $3.29 \pm 0.03 \mathrm{fg}$ & $1.54 \pm 0.02 \mathrm{~b}$ & $4.80 \pm 0.02 \mathrm{~d}$ & $3.17 \pm 0.02 \mathrm{c}$ & $4.47 \pm 0.02 \mathrm{c}$ \\
\hline 27 & 35 & 25 & 120 & $7.35 \pm 0.02 \mathrm{k}$ & $2.29 \pm 0.02 \mathrm{~b}$ & $3.19 \pm 0.02 \mathrm{~h}$ & $5.88 \pm 0.08 \mathrm{fg}$ & $3.26 \pm 0.02 \mathrm{gh}$ & $1.22 \pm 0.02 \mathrm{~b}$ & $4.24 \pm 0.03 \mathrm{i}$ & $2.23 \pm 0.02 \mathrm{k}$ & $3.83 \pm 0.03 \mathrm{i}$ \\
\hline
\end{tabular}


Production and Nutritional Evaluation of Instantized Kunun Gyada, a Traditional Nigerian ..

Table 5. Experimental Design and Observed Mean values of Non-Essential Amino acid Profile of Extrudates (g/100g Protein)

\begin{tabular}{|c|c|c|c|c|c|c|c|c|c|c|c|}
\hline \multirow[b]{2}{*}{ Design points } & \multicolumn{5}{|c|}{ Independent variables } & \multicolumn{6}{|c|}{ Values in their natural form } \\
\hline & $x_{1}$ & $x_{2}$ & $x_{3}$ & Alanine & Aspartic acid & Cystine & Glutamic acid & Glycine & Proline & Serine & Tyrosine \\
\hline 1 & 15 & 15 & 100 & $3.51 \pm 0.02 f$ & $9.84 \pm 0.04 \mathrm{~b}$ & $1.09 \pm 0.03 \mathrm{~b}$ & $13.36 \pm 0.03 \mathrm{~m}$ & $3.58 \pm 0.03 j$ & $3.28 \pm 0.02 \mathrm{~h}$ & $3.01 \pm 0.03 \mid$ & $2.89 \pm 0.03 \mathrm{j}$ \\
\hline 2 & 15 & 15 & 110 & $3,47 \pm 0.02 f$ & $9.93 \pm 0.06 \mathrm{~b}$ & $1.07 \pm 0.03 \mathrm{~b}$ & $13.25 \pm 0.05 n$ & $3.44 \pm 0.02 \mathrm{k}$ & $3.07 \pm 0.02 \mathrm{ij}$ & $2.96 \pm 0.02 \mathrm{~m}$ & $2.86 \pm 0.01 \mathrm{jk}$ \\
\hline 3 & 15 & 15 & 120 & $3.37 \pm 0.01 \mathrm{~h}$ & $9.87 \pm 0.03 \mathrm{~b}$ & $1.07 \pm 0.02 b$ & $12.88 \pm 0.03 p$ & $3.36 \pm 0.031$ & $2.84 \pm 0.04 i$ & $2.95 \pm 0.02 \mathrm{mn}$ & $2.83 \pm 0.02 \mathrm{k}$ \\
\hline 4 & 15 & 20 & 100 & $3.26 \pm 0.06 i$ & $9.75 \pm 0.02 b$ & $1.08 \pm 0.03 \mathrm{~b}$ & $12.75 \pm 0.03 q$ & $3.35 \pm 0.011$ & $2.45 \pm 0.04 n$ & $2.88 \pm 0.07 \mathrm{op}$ & $2.76 \pm 0.021$ \\
\hline 5 & 15 & 20 & 110 & $3.22 \pm 0.03 \mathrm{j}$ & $9.58 \pm 0.03 \mathrm{~b}$ & $1.02 \pm 0.03 \mathrm{~b}$ & $12.21 \pm 0.02 r$ & $3.36 \pm 0.041$ & $1.99 \pm 0.06 q$ & $2.87 \pm 0.05$ op & $2.74 \pm 0.041$ \\
\hline 6 & 15 & 20 & 120 & $3.20 \pm 0.01 \mathrm{jk}$ & $9.49 \pm 0.02 b$ & $0.98 \pm 0.01 b$ & $12.03 \pm 0.045$ & $3.34 \pm 0.021$ & $2.08 \pm 0.03 p$ & $2.79 \pm 0.02 q$ & $2.57 \pm 0.02 \mathrm{~m}$ \\
\hline 7 & 15 & 25 & 100 & $3.16 \pm 0.01 \mathrm{k}$ & $9.14 \pm 0.07 b$ & $0.94 \pm 0.02 b$ & $11.87 \pm 0.02 t$ & $3.23 \pm 0.02 n$ & $2.33 \pm 0.050$ & $2.65 \pm 0.02 r$ & $2.52 \pm 0.01 n$ \\
\hline 8 & 15 & 25 & 110 & $3.11 \pm 0.02 \mathrm{~m}$ & $8.96 \pm 0.02 b$ & $0.91 \pm 0.03 \mathrm{~b}$ & $11.03 \pm 0.05 u$ & $3.13 \pm 0.030$ & $2.61 \pm 0.03 \mathrm{~m}$ & $2.59 \pm 0.03 \mathrm{~s}$ & $2.47 \pm 0.020$ \\
\hline 9 & 15 & 25 & 120 & $2.98 \pm 0.030$ & $8.89 \pm 0.02 b$ & $0.98 \pm 0.05 b$ & $10.96 \pm 0.04 v$ & $3.06 \pm 0.03 p$ & $2.87 \pm 0.021$ & $2.57 \pm 0.025$ & $2.34 \pm 0.04 p$ \\
\hline 10 & 25 & 15 & 100 & $3.89 \pm 0.02 \mathrm{c}$ & $11.26 \pm 0.03 b$ & $1.62 \pm 0.03 \mathrm{~b}$ & $16.38 \pm 0.02 f$ & $4.36 \pm 0.04 c$ & $3.37 \pm 0.02 b$ & $3.96 \pm 0.04 d$ & $3.51 \pm 0.03 b$ \\
\hline 11 & 25 & 15 & 110 & $3.87 \pm 0.02 \mathrm{c}$ & $11.18 \pm 0.03 b$ & $1.54 \pm 0.07 \mathrm{~b}$ & $16.09 \pm 0.03 \mathrm{~g}$ & $4.19 \pm 0.02 \mathrm{e}$ & $3.84 \pm 0.02 b$ & $3.93 \pm 0.02 \mathrm{de}$ & $3.41 \pm 0.02+d$ \\
\hline 12 & 25 & 15 & 120 & $3.66 \pm 0.02 \mathrm{~d}$ & $10.87 \pm 0.02 b$ & $1.35 \pm 0.03 \mathrm{~b}$ & $15.56 \pm 0.04 i$ & $3.88 \pm 0.04 \mathrm{~h}$ & $3.42 \pm 0.04 f$ & $3.91 \pm 0.02 \mathrm{ef}$ & $3.06 \pm 0.02 \mathrm{~h}$ \\
\hline 13 & 25 & 20 & 100 & $3.47 \pm 0.02 \mathrm{~g}$ & $3.47 \pm 0.04 a$ & $1.32 \pm 0.001 \mathrm{~b}$ & $14.35 \pm 0.02 j$ & $3.55 \pm 0.03 \mathrm{j}$ & $3.08 \pm 0.03 \mathrm{i}$ & $3.88 \pm 0.01 \mathrm{f}$ & $2.87 \pm 0.02 j$ \\
\hline 14 & 25 & 20 & 110 & $3.21 \pm 0.03 \mathrm{j}$ & $9.16 \pm 0.04 b$ & $1.22 \pm 0.03 \mathrm{~b}$ & $12.88 \pm 0.01 \mathrm{p}$ & $3.25 \pm 0.03 \mathrm{mn}$ & $2.88 \pm 0.031$ & $2.86 \pm 0.03 p$ & $2.74 \pm 0.04 i$ \\
\hline 15 & 25 & 20 & 120 & $3.18 \pm 0.01 \mathrm{jk}$ & $9.23 \pm 0.02 b$ & $1.31 \pm 0.02 \mathrm{~b}$ & $13.07 \pm 0.020$ & $3.28 \pm 0.02 \mathrm{~m}$ & $2.94 \pm 0.02 \mathrm{k}$ & $2.91 \pm 0.02 \mathrm{no}$ & $2.73 \pm 0.021$ \\
\hline 16 & 25 & 25 & 100 & $3.13 \pm 0.021 \mathrm{~m}$ & $9.27 \pm 0.01 \mathrm{~b}$ & $1.58 \pm 0.01 \mathrm{~b}$ & $13.45 \pm 0.03 \mid$ & $3.34 \pm 0.021$ & $2.94 \pm 0.02 \mathrm{k}$ & $3.11 \pm 0.03 \mathrm{k}$ & $2.86 \pm 0.03 \mathrm{jk}$ \\
\hline 17 & 25 & 25 & 110 & $3.11 \pm 0.02 \mathrm{~m}$ & $9.31 \pm 0.02 b$ & $1.67 \pm 0.02 \mathrm{~b}$ & $13.97 \pm 0.02 \mathrm{k}$ & $3.48 \pm 0.01 \mathrm{k}$ & $3.03 \pm 0.02 j$ & $3.25 \pm 0.02 j$ & $2.97 \pm 0.02 i$ \\
\hline 18 & 25 & 25 & 120 & $3.06 \pm 0.02 \mathrm{n}$ & $9.34 \pm 0.04 \mathrm{~b}$ & $1.75 \pm 0.03 \mathrm{~b}$ & $14.34 \pm 0.03 \mathrm{j}$ & $3.57 \pm 0.03 \mathrm{j}$ & $3.07 \pm 0.02 i j$ & $3.34 \pm 0.03 \mathrm{i}$ & $3.05 \pm 0.03 \mathrm{~h}$ \\
\hline 19 & 35 & 15 & 100 & $3.57 \pm 0.02 \mathrm{e}$ & $10.76 \pm 0.03 \mathrm{~b}$ & $2.07 \pm 0.02 \mathrm{~b}$ & $16.84 \pm 0.03 d$ & $3.96 \pm 0.02 \mathrm{~g}$ & $3.67 \pm 0.02 d$ & $3.93 \pm 0.03 \mathrm{~g}$ & $3.36 \pm 0.03 \mathrm{ef}$ \\
\hline 20 & 35 & 15 & 110 & $3.65 \pm 0.02 d$ & $10.96 \pm 0.03 b$ & $2.17 \pm 0.02 b$ & $17.07 \pm 0.01 \mathrm{c}$ & $4.07 \pm 0.04 f$ & $3.77 \pm 0.02 c$ & $4.06 \pm 0.05 c$ & $3.39 \pm 0.02 \mathrm{de}$ \\
\hline 21 & 35 & 15 & 120 & $3.98 \pm 0.01 \mathrm{~b}$ & $11.70 \pm 0.03 b$ & $2.28 \pm 0.02 \mathrm{~b}$ & $17.18 \pm 0.05 b$ & $4.31 \pm 0.03 d$ & $3.88 \pm 0.02 b$ & $4.18 \pm 0.01 \mathrm{~b}$ & $3.46 \pm 0.02 \mathrm{c}$ \\
\hline 22 & 35 & 20 & 100 & $4.03 \pm 0.03 a$ & $11.69 \pm 0.50 \mathrm{~b}$ & $2.41 \pm 0.04 \mathrm{~b}$ & $17.25 \pm 0.03 a$ & $4.46 \pm 0.03 b$ & $4.07 \pm 0.02 \mathrm{a}$ & $4.21 \pm 0.02 \mathrm{~b}$ & $3.53 \pm 0.02 b$ \\
\hline 23 & 35 & 20 & 110 & $4.05 \pm 0.03 a$ & $12.30 \pm 0.03 b$ & $2.40 \pm 0.02 \mathrm{~b}$ & $17.28 \pm 0.03 a$ & $4.57 \pm 0.01 \mathrm{a}$ & $4.09 \pm 0.02 \mathrm{a}$ & $4.26 \pm 0.03 \mathrm{a}$ & $3.67 \pm 0.02 a$ \\
\hline 24 & 35 & 20 & 120 & $3.97 \pm 0.03 \mathrm{~b}$ & $11.99 \pm 0.02 b$ & $2.04 \pm 0.01 \mathrm{~b}$ & $17.06 \pm 0.02 c$ & $4.27 \pm 0.02 \mathrm{~d}$ & $3.88 \pm 0.04 \mathrm{~b}$ & $4.21 \pm 0.02 \mathrm{~b}$ & $3.53 \pm 0.02 b$ \\
\hline 25 & 35 & 25 & 100 & $3.65 \pm 0.02 \mathrm{~d}$ & $11.65 \pm 0.03 b$ & $8.53 \pm 11.55 \mathrm{a}$ & $16.75 \pm 0.03 \mathrm{e}$ & $4.08 \pm 0.04 f$ & $3.55 \pm 0.03 \mathrm{e}$ & $3.87 \pm 0.02 \mathrm{fg}$ & $3.34 \pm 0.03 f$ \\
\hline 26 & 35 & 25 & 110 & $3.52 \pm 0.02 f$ & $11.98 \pm 0.01 \mathrm{~b}$ & $1.56 \pm 0.03 \mathrm{~b}$ & $15.78 \pm 0.02 \mathrm{~h}$ & $3.96 \pm 0.02 \mathrm{~g}$ & $3.42 \pm 0.01 \mathrm{f}$ & $3.56 \pm 0.02 \mathrm{~h}$ & $3.19 \pm 0.07 \mathrm{~g}$ \\
\hline 27 & 35 & 25 & 120 & $3.46 \pm 0.04 \mathrm{~g}$ & $10.07 \pm 0.02 b$ & $1.35 \pm 0.03 \mathrm{~b}$ & $14.36 \pm 0.04 j$ & $3.80 \pm 0.03 \mathrm{i}$ & $3.37 \pm 0.02 \mathrm{~g}$ & $3.24 \pm 0.03 \mathrm{j}$ & $3.05 \pm 0 . h$ \\
\hline
\end{tabular}

\section{Amino Acids Profile of Extrudates}

The results of both essential and non-essential amino acids are shown in Tables 4 and 5 respectively. All the amino acids showed highest value at design point number 23 with extrusion parameters $35 \%$ feed composition, $20 \%$ feed moisture content, and $110^{\circ} \mathrm{C}$ barrel temperature which also has high general acceptability value of 6.4 . Among all the amino acids at extrusion parameters $35 \%, 20 \%, 110^{\circ} \mathrm{C}$, Methionine had the lowest value of $1.63 \mathrm{~g}$ and Glutamic acid has the highest value of $17.28 \mathrm{~g}$. From essential amino acids, Methionine had the lowest value of $1.63 \mathrm{~g}$, Leucine had the highest value of $7.34 \mathrm{~g}$ and lysine had $3.54 \mathrm{~g}$ at the same design point $23\left(35 \% 20 \% 110^{\circ} \mathrm{C}\right)$. In non-essential amino acids, Cystine had the lowest value of $2.40 \mathrm{~g}$ and Glutamic acid had the highest value of $17.28 \mathrm{~g}$ at $35 \% 20 \%$

\section{Discussion}

Proximate Compositions of Raw Materials and Extrudates

The results of the proximate composition of raw and extruded samples are shown in Tables 4 and 5 . The high crude protein content of the roasted groundnut paste may have accounted for the increase in the crude protein content of extrudates; the linear increase in protein content of the extruded sample was also reported for Fura as a result of increase in the amount of cowpea flour from 15 to $35 \%$ (Filli et al., 2011). The decrease in the fat content may be as a result of its use as an energy source during germination and/or utilization by microorganisms during soaking and germination (Chavan and Khadam, 1989b). The low carbohydrate content of malted sorghum flour, $74.3 \%$, could be due to gradual degradation of starch by enzymes during germination. Soaking and germination have been reported to enhance the lost of starch (Chavan and Khadam, 1989a).The higher ash content $3 \%$ could be attributed to loss of dry matter, especially carbohydrates, through respiration during sprouting (Chavan and Khadams, 1989a). The variation in the moisture content, $11.1 \%$ to $11.21 \%$ observed in the unmalted sorghum flour and the malted flour samples could be as a result of pre-processing methods such as soaking, germination, drying and storage conditions. Moisture content of malted sorghum flour was higher than that of the extrudates and roasted groundnuts paste. The result agrees with the work of Badrie and Mellowes (1991) who reported that increasing extrusion temperature from $105^{\circ} \mathrm{C}$ to $150^{\circ} \mathrm{C}$ reduced moisture level in extrudates.

\section{Amino Acids Profile of Extrudates}

All amino acids of extrudates increased with increase in feed composition and decreased with increase in both feed moisture content and barrel temperature.

\section{Conclusion}

Germination and extrusion cooking of cereal grains and legumes have been shown to be effective in improving their nutritive value (Chavan and Khadams, 1989a; 1989b). The result of proximate mean composition of extrudates indicates increase in nutrients which may be as a result of the increase in roasted groundnuts paste, use of malted sorghum flour, and the application of extrusion cooking which improve the product quality. 


\section{Reference}

[1]. Association of Official Analytical Chemists (AOAC) (2000). Official Methods of Analysis, 17 ${ }^{\text {th }}$ Edition Gaithersburg, Maryland, USA.

[2]. Gibson, R.S., Yeudallf, Drost N., Mitimuni B., Culliman T., (1998). Dietary intervention to prevent Zinc difficiency. American Clinical Nutrition 68: 4845 - 4875 .

[3]. Food and Agricultural Organization (FAO) 2015

[4]. Zhang, G; and Hamaker, B. R. (1998). Low x-Amylase digestibility of cooked Sorghum flours and the effect of protein. Journal of Cereal Chemistry. 75: $710-713$

[5]. United States Department of Agriculture, (2015). World Agricultural Production. Retrieved 25/06/2015 from http://www.fas.qov/wap/current

[6]. Eke-Ejiofor J., Klin-Kabari D.B., Chukwu E.C., (2012). Effect of processing methods on the proximate, mineral and fungi properties of groundnuts (Arachis Hypogaea). Seed Journal of Agricultural and Biological Science 3: 257-261.

[7]. Castells M., Marin S., Sanchis V., Ramos A.J., (2005). Fate of micotoxin in cereal during extrusion cooking. A Review Food Additional Container. 22: 150-157. Doi 10. 1080/0265203050. 0037969 (Pub med)

[8]. Guy ,R., (2001). Extrusion cooking and applications. Woodhead Publishing limited Cambridge, England Plahar, W.A., Onowa Okezie, B., Gyato, C. (2003). Development of high protein weaning foods by extrusion cooking using peanut, maize and soybeans. Plant Food Human Nutrition 58: 58-61.

[9]. Eastman, J., Orthoefer, F. and Solorio, S. (2001). Using extrusion to create breakfast Cereal products. Cereal Foods World, 46: 468471.

[10]. Elkhalita, A.E.O. and Benhardt, A. (2010). Influence of grain germination on functional properties of Sorghum flour, Food Chemistry, 121: 387-392.

[11]. Filli K. B., Nkam I., Abubakar U. M., and Jideani V. A. (2010). Influence of extrusion variables on some functional properties of extruded millet-soybean for the manufacture of "Fura": A Nigerian traditional food.

[12]. Filli K.B., Nkama I., Jideani V.A. and Abubakar U.M., (2011). Application of response surface methodology for the study of composition of extruded millet cow-pea mixtures for the manufacture of fura: a Nigerian food, African Journal of Food Science vol.5 (16).

[13]. Blamire J. Science @ a distance @ (2003). Retrieved August, 16 ${ }^{\text {th }}$ 2014. www. Brooklyn. Cuny. Edu./bcahp/50KC/C....

[14]. Benitez, L. V. (1989). Amino Acid and fatty acid profiles in aquaculture nutrition studies, p. 23-35. In S. S. De SILVER (ed.) Fish Nutrition Research in Asia. Society Special Publication. 4. 166 p. Asian Fisheries Society, Manila Philipines.

[15]. Charvan J.K, and Khadam, S.S., (1989a). Nutritional improvement of Cereals by sprouting. Critical Review in Food Science and Nutrition 28: 401-437.

[16]. Badrie N. and Mellowes W.A., (1991). Texture and microstructure of cassava flour extrudates. Journal of Food Science. 56 (1): $1319-22$ 